ANNALES

POLONICI MATHEMATICI

$84.2(2004)$

\title{
Proper holomorphic self-mappings of the symmetrized bidisc
}

\author{
by ARmen Edigarian (Kraków)
}

\begin{abstract}
We characterize proper holomorphic self-mappings $\mathbb{G}_{2} \rightarrow \mathbb{G}_{2}$ for the symmetrized bidisc $\mathbb{G}_{2}=\left\{\left(\lambda_{1}+\lambda_{2}, \lambda_{1} \lambda_{2}\right):\left|\lambda_{1}\right|,\left|\lambda_{2}\right|<1\right\} \subset \mathbb{C}^{2}$.
\end{abstract}

Let $\mathbb{D}$ be the unit disc. Set

$$
\pi: \mathbb{C}^{2} \ni\left(\lambda_{1}, \lambda_{2}\right) \mapsto\left(\lambda_{1}+\lambda_{2}, \lambda_{1} \lambda_{2}\right) \in \mathbb{C}^{2}
$$

and $\mathbb{G}_{2}=\pi\left(\mathbb{D}^{2}\right)$. The domain $\mathbb{G}_{2}$ is called the symmetrized bidisc. It has recently been studied by many authors, e.g. [1], [7], [10], [3], [4]. The original motivation for the study of the complex geometry of the symmetrized bidisc comes from control engineering [2].

From the complex analysis point of view the symmetrized bidisc is important since it is the first known example of a bounded pseudoconvex domain for which the Carathéodory and Lempert functions coincide, but which cannot be exhausted by domains biholomorphic to convex ones (see [6], [3], [4]).

Recall that a mapping $f: X \rightarrow Y$ between topological spaces $X, Y$ is called proper if $f^{-1}(K)$ is a compact subset of $X$ for any compact set $K \subset Y$. The main purpose of the paper is to give the following characterization of proper holomorphic self-mappings of the symmetrized bidisc.

TheOREM 1. Let $f: \mathbb{G}_{2} \rightarrow \mathbb{G}_{2}$ be a proper holomorphic mapping. Then there exists a finite Blaschke product $B$ such that

$$
f\left(\pi\left(\lambda_{1}, \lambda_{2}\right)\right)=\pi\left(B\left(\lambda_{1}\right), B\left(\lambda_{2}\right)\right)
$$

for any $\lambda_{1}, \lambda_{2} \in \mathbb{D}$.

2000 Mathematics Subject Classification: Primary 32H35.

Key words and phrases: proper holomorphic mapping, symmetrized bidisc, Blaschke product.

The author was supported in part by the KBN grant No. 5 P03A 03321. 
By a (finite) Blaschke product we mean a function of the form

$$
B(\lambda)=e^{i \tau} \prod_{j=1}^{m} \frac{\lambda-a_{j}}{1-\bar{a}_{j} \lambda}
$$

where $\tau \in \mathbb{R}, m \in \mathbb{N}$ and $a_{1}, \ldots, a_{m} \in \mathbb{D}$. Recall that a holomorphic function $g: \mathbb{D} \rightarrow \mathbb{D}$ is proper if and only if it is a finite Blaschke product.

Theorem 1 implies that if $f$ is an automorphism then $f\left(\pi\left(\lambda_{1}, \lambda_{2}\right)\right)=$ $\pi\left(h\left(\lambda_{1}\right), h\left(\lambda_{2}\right)\right)$, where $h$ is an automorphism of the unit disc $\mathbb{D}$ (see [7]).

The above result is a corollary of the following:

Theorem 2. Let $f: \mathbb{D}^{2} \rightarrow \mathbb{G}_{2}$ be a proper holomorphic mapping. Then there exist finite Blaschke products $B_{1}, B_{2}$ such that

$$
f\left(\lambda_{1}, \lambda_{2}\right)=\left(B_{1}\left(\lambda_{1}\right)+B_{2}\left(\lambda_{2}\right), B_{1}\left(\lambda_{1}\right) B_{2}\left(\lambda_{2}\right)\right),
$$

for any $\lambda_{1}, \lambda_{2} \in \mathbb{D}$.

Note that $\pi: \mathbb{C}^{2} \rightarrow \mathbb{C}^{2}$ is a proper holomorphic mapping and the singular set is equal to $\Sigma_{2}=\pi(\Delta)$, where $\Delta=\{(\lambda, \lambda): \lambda \in \mathbb{C}\}$, i.e. $\pi: \mathbb{C}^{2} \backslash \Delta \rightarrow$ $\mathbb{C}^{2} \backslash \Sigma_{2}$ is a holomorphic covering.

Let us first gather some elementary properties of the symmetrized bidisc (see e.g. [1]).

Proposition 3. (1) $(s, p) \in \mathbb{G}_{2}$ if and only if $|s-\bar{s} p|+|p|^{2}<1$;

(2) if $(s, p) \in \partial \mathbb{G}_{2}$ then $|s-\bar{s} p|+|p|^{2}=1$;

(3) $\pi^{-1}\left(\mathbb{G}_{2}\right)=\mathbb{D}^{2}$;

(4) $\pi^{-1}\left(\partial \mathbb{G}_{2}\right)=\partial\left(\mathbb{D}^{2}\right)$;

(5) $\Sigma_{2} \cap \partial \mathbb{G}_{2}=\left\{\left(2 \lambda, \lambda^{2}\right):|\lambda|=1\right\}$.

Lemma 4. Assume that $\varphi: \mathbb{D} \rightarrow \partial \mathbb{G}_{2}$ is a holomorphic mapping. Then there exist $a \theta \in \mathbb{R}$ and a holomorphic function $\psi: \mathbb{D} \rightarrow \overline{\mathbb{D}}$ such that $\varphi(\lambda)=\left(e^{i \theta}+\psi(\lambda), e^{i \theta} \psi(\lambda)\right)$ for any $\lambda \in \mathbb{D}$.

Proof. We know that $\partial \mathbb{G}_{2} \subset\left\{(s, p):|s-\bar{s} p|+|p|^{2}=1\right\}$. If $\varphi\left(\lambda_{0}\right) \in$ $\Sigma_{2} \cap \partial \mathbb{G}_{2}$ for some $\lambda_{0} \in \mathbb{D}$ then $\varphi_{2}=e^{i \tau}$ for some $\tau \in \mathbb{R}$ and therefore $\varphi_{1}=$ const.

So, assume that $\varphi(\mathbb{D}) \subset \mathbb{C}^{2} \backslash \Sigma_{2}$. Hence, there exists a holomorphic mapping $\widetilde{\varphi}: \mathbb{D} \rightarrow \mathbb{C}^{2}$ such that $\varphi=\pi \circ \widetilde{\varphi}$. Now, it suffices to note that $\widetilde{\varphi}: \mathbb{D} \rightarrow \partial\left(\mathbb{D}^{2}\right)$.

Proof of Theorem 2. We use similar methods to those in the proof of the Remmert-Stein theorem (see e.g. [9, p. 71]).

Let $f=\left(f_{1}, f_{2}\right)$. Assume that $\mathbb{D} \ni w_{\nu} \rightarrow w_{0} \in \partial \mathbb{D}, \nu \in \mathbb{N}$, is any sequence. The functions $\varphi_{j \nu}(z)=f_{j}\left(z, w_{\nu}\right), j \in\{1,2\}, \nu \geq 1$, are holomorphic in $\mathbb{D}$. By Montel's theorem there is a subsequence $\left\{\nu_{k}\right\}$ so that $\varphi_{j \nu_{k}} \rightarrow \varphi_{j}$ uniformly on compact subsets of $\mathbb{D}$. Moreover, $\left(\varphi_{1}(z), \varphi_{2}(z)\right) \in \partial \mathbb{G}_{2}$ for any $z \in \mathbb{D}$ (here we use the properness of $f$ ). 
By Lemma 4, $\varphi_{1}=e^{i \theta}+\psi$ and $\varphi_{2}=e^{i \theta} \psi$. Now, by the Weierstrass theorem,

$$
\begin{array}{ll}
\frac{\partial f_{1}\left(z, w_{\nu_{k}}\right)}{\partial z} \rightarrow \psi^{\prime}(z), & \frac{\partial^{2} f_{1}\left(z, w_{\nu_{k}}\right)}{\partial z^{2}} \rightarrow \psi^{\prime \prime}(z), \\
\frac{\partial f_{2}\left(z, w_{\nu_{k}}\right)}{\partial z} \rightarrow e^{i \theta} \psi^{\prime}(z), & \frac{\partial^{2} f_{2}\left(z, w_{\nu_{k}}\right)}{\partial z^{2}} \rightarrow e^{i \theta} \psi^{\prime \prime}(z) .
\end{array}
$$

Set

$$
\begin{aligned}
H_{1}(z, w)= & \frac{\partial f_{1}(z, w)}{\partial z} \frac{\partial^{2} f_{2}(z, w)}{\partial z^{2}}-\frac{\partial^{2} f_{1}(z, w)}{\partial z^{2}} \frac{\partial f_{2}(z, w)}{\partial z} \\
H_{2}(z, w)= & f_{2}(z, w)\left(\frac{\partial f_{1}(z, w)}{\partial z}\right)^{2}+\left(\frac{\partial f_{2}(z, w)}{\partial z}\right)^{2} \\
& -f_{1}(z, w) \frac{\partial f_{1}(z, w)}{\partial z} \frac{\partial f_{2}(z, w)}{\partial z} .
\end{aligned}
$$

From (4) and (5) we get

$$
H_{1}\left(z, w_{\nu_{k}}\right) \rightarrow 0, \quad H_{2}\left(z, w_{\nu_{k}}\right) \rightarrow 0 .
$$

Hence, $H_{1}(z, w) \equiv 0$ and $H_{2}(z, w) \equiv 0$.

Set $A=\left\{(z, w) \in \mathbb{D}^{2}: \frac{\partial f_{1}}{\partial z}(z, w)=0\right\}$. Note that $A$ is a proper analytic subset of $\mathbb{D}^{2}$. Indeed, if $A=\mathbb{D}^{2}$ then the function $\psi$ in (4) is identically zero, so from (5) we have $\partial f_{2} / \partial z \equiv 0$. Hence, $f_{1}(z, w)=g_{1}(w)$ and $f_{2}(z, w)=$ $g_{2}(w)$ for $(z, w) \in \mathbb{D}^{2}$, where $g_{1}, g_{2}$ are holomorphic functions on $\mathbb{D}$. This contradicts the properness of $f$ (for a fixed $w \in \mathbb{D}$ take $z \rightarrow \partial \mathbb{D}$ ).

Note that $\mathbb{D}^{2} \backslash A$ is a domain (i.e. an open connected set). By (6) there exists a holomorphic function $g_{1}$ such that

$$
\frac{\partial f_{2}(z, w)}{\partial z}=g_{1}(w) \frac{\partial f_{1}(z, w)}{\partial z}
$$

on $\mathbb{D}^{2} \backslash A$. From (7) we get

$$
f_{2}(z, w)=g_{1}(w) f_{1}(z, w)-g_{1}^{2}(w)
$$

for $(z, w) \in \mathbb{D}^{2} \backslash A$. Note that $f(z, w)=\pi\left(g_{1}(w), f_{1}(z, w)-g_{1}(w)\right)$. So, $\widetilde{f}(z, w)=\left(g_{1}(w), f_{1}(z, w)-g_{1}(w)\right)$ is a holomorphic mapping $\mathbb{D}^{2} \backslash A \rightarrow \mathbb{D}^{2}$. Since $g_{1}$ is bounded on $\mathbb{D}^{2} \backslash A$, it extends holomorphically to $\mathbb{D}^{2}$. So, $f=\pi \circ \tilde{f}$ where $\widetilde{f}=\left(g_{1}, f_{1}-g_{1}\right)$.

Repeating similar arguments for $w$ we show that there exists a holomorphic mapping $g_{2}$ such that

$$
f_{2}(z, w)=g_{2}(z) f_{1}(z, w)-g_{2}^{2}(z) .
$$

From (10) and (11) we get $f_{1}(z, w)=g_{1}(w)+g_{2}(z)$ and $f_{2}(z, w)=g_{1}(w) g_{2}(z)$. Now, it suffices to note that $g_{1}, g_{2}: \mathbb{D} \rightarrow \mathbb{D}$ are proper holomorphic functions and, therefore, they are finite Blaschke products. 
REMARK 5. Note that one may consider proper holomorphic self-mappings of the symmetrized polydisc (see e.g. [8]). In [5], we will show that they have a similar description.

\section{References}

[1] J. Agler and N. J. Young, A Schwarz lemma for the symmetrized bidisc, Bull. London Math. Soc. 33 (2001), 175-186.

[2] - - - The hyperbolic geometry of the symmetrized bidisc, preprint, 2003.

[3] C. Costara, The symmetrized bidisc as a counterexample to the converse of Lempert's theorem, Bull. London Math. Soc., to appear.

[4] A. Edigarian, A note on Costara's paper, Ann. Math. Polon. 83 (2004), 189-191.

[5] A. Edigarian and W. Zwonek, Proper holomorphic self-mappings of the symmetrized polydisc, Arch. Math. (Basel), to appear.

[6] M. Jarnicki and P. Pflug, Invariant Distances and Metrics in Complex Analysis, de Gruyter Exp. Math. 9, de Gruyter, 1993.

[7] -, 一, On automorphisms of the symmetrized bidisc, preprint, 2003.

[8] -, - Invariant distances and metrics in complex analysis-revisited, Dissertationes Math., to appear.

[9] R. Narasimhan, Several Complex Variables, Chicago Lectures in Math., Univ. of Chicago Press, 1971.

[10] P. Pflug and W. Zwonek, Description of all complex geodesics in the symmetrized bidisc, preprint, 2003.

Institute of Mathematics

Jagiellonian University

Reymonta 4/526

30-059 Kraków, Poland

E-mail: Armen.Edigarian@im.uj.edu.pl 